\title{
Avogadro constant challenge
}

\author{
William B. Jensen • Juris Meija
}

Published online: 5 March 2010

(C) Crown copyright in right of Canada 2010

We would like to invite you to participate in the Analytical Challenge, a series of puzzles to entertain and challenge our readers. This special $A B C$ feature has established itself as a truly unique quiz series, with a new scientific puzzle published every other month. Readers can access the complete collection of published problems with their solutions on the Analytical and Bioanalytical Chemistry homepage at http://www.springer.com/abc. Test your knowledge and tease your wits in diverse areas of analytical and bioanalytical chemistry by viewing this collection.

In the present challenge Avogadro constant is the topic. And please note that there is a prize to be won (a Springer book of your choice up to a value of $€$ 75). Please read on...

\section{Meet the Avogadro constant challenge}

If the atomic weight of a hydrogen molecule is two, then how many atoms are there in two grams of hydrogen? The answer to that is the Avogadro constant - the conversion factor between the microscopic (atomic) and macroscopic weight scales. A far more general importance of the

W. B. Jensen

Department of Chemistry, University of Cincinnati,

Cincinnati, OH 45221-0172, USA

J. Meija ( $\bowtie)$

Institute for National Measurement Standards,

National Research Council Canada,

1200 Montreal Road,

Ottawa, ON K1A 0R6, Canada

e-mail: juris.meija@nrc.ca
Avogadro constant is that it provides a link between the properties of individual molecules and the properties of bulk matter; for example, it links the energy of individual molecules, which can be determined by spectroscopy, to the energy of bulk matter measured in calorimetric experiments.

The exact value of the Avogadro constant is a rather controversial topic $d u$ jour. Significant momentum currently exists to modernize the International System of Units (SI) $[1,2]$. It is foreseen that in the near future new definitions of the kilogram and mole will be based on fixed values for the Planck and Avogadro constants; much like the meter was defined in 1983 by fixing the value for the speed of light in vacuum (note that the Planck constant is related to the Avogadro constant). However, recent measurement results of the Avogadro constant are not in agreement: watt balance experiments suggest a value of $6.0221418(3)$ [3] or $6.0221400(4) \times 10^{23} \mathrm{~mol}^{-1}$ [4], whereas silicon sphere experiments give significantly lower value, $6.022135(2) \times 10^{23} \mathrm{~mol}^{-1}[5,6]$. The importance of this, of course, is that any new definition of the kilogram or the mole must wait until the present inconsistency between the values of Avogadro constant is formally resolved.

\section{The challenge}

Sometime amid the heights of the Cold War, the accepted value of the Avogadro constant suddenly changed in the third digit! This was quite a change, considering that there is currently a lingering controversy regarding the discrepancy in the sixth digit. Can you explain the sudden decrease in Avogadro constant during the Cold War? 


\section{References}

1. Milton MJT, Williams JM, Bennett SJ (2007) Metrologia 44:356-364

2. Williams E (2007) IEEE Trans Instrum Meas 56:646-650

3. Mohr PJ (2008) Rev Mod Phys 80:633-730

4. Robinson IA, Kibble BP (2007) Metrologia 44:427-440

5. Fujii K, Waseda A, Kuramoto N, Mizushima S, Becker P, Bettin H, Nicolaus A, Kuetgens U, Valkiers S, Taylor P, De Bievre P, Mana G, Massa E, Matyi R, Kessler EG, Hanke M (2005) IEEE Trans Instrum Meas 54:854-859

6. Eichenberger A, Geneves G, Gournay P (2009) Eur Phys J Special Topics 172:363-383

We invite our readers to participate in the Analytical Challenge by solving the puzzle above. Please send the correct solution to abc-challenge@springer.comby June 20, 2010. Make sure you enter "Avogadro constant challenge" in the subject line of your e-mail. The winner will be notified by e-mail and his/her name will be published on the 'Analytical and Bioanalytical Chemistry' website at http://www.springer.com/abc and in the journal. Readers will find the solution and a short explanation on the 'Analytical and Bioanalytical Chemistry' website after June 20, 2010, and in the journal (Issue 398/1).

The next Analytical Challenge will be published in Issue 397/5, July 2010. If you have enjoyed solving this Challenge you are invited to try the previous puzzles on the 'Analytical and Bioanalytical Chemistry' website. 